\title{
Staphylococcus aureus ST398 gene expression profiling during ex vivo colonization of porcine nasal epithelium
}

Pawel Tulinski ${ }^{1,5}$, Birgitta Duim ${ }^{1,7^{*}}$, Floyd R Wittink ${ }^{2,6}$, Martijs J Jonker ${ }^{2}$, Timo M Breit ${ }^{2}$, Jos P van Putten ${ }^{1}$, Jaap A Wagenaar ${ }^{1,3}$ and Ad C Fluit ${ }^{4}$

\begin{abstract}
Background: Staphylococcus aureus is a common human and animal opportunistic pathogen. In humans nasal carriage of S. aureus is a risk factor for various infections. Methicillin-resistant S. aureus ST398 is highly prevalent in pigs in Europe and North America. The mechanism of successful pig colonization by MRSA ST398 is poorly understood. Previously, we developed a nasal colonization model of porcine nasal mucosa explants to identify molecular traits involved in nasal MRSA colonization of pigs.

Results: We report the analysis of changes in the transcription of MRSA ST398 strain S0462 during colonization on the explant epithelium. Major regulated genes were encoding metabolic processes and regulation of these genes may represent metabolic adaptation to nasal mucosa explants. Colonization was not accompanied by significant changes in transcripts of the main virulence associated genes or known human colonization factors. Here, we documented regulation of two genes which have potential influence on S. aureus colonization; cysteine extracellular proteinase (sсpA) and von Willebrand factor-binding protein (vWbp, encoded on SaPlbov5). Colonization with isogenic-deletion strains ( $\triangle v w b p$ and $\triangle s c p A$ ) did not alter the ex vivo nasal S. aureus colonization compared to wild type.
\end{abstract}

Conclusions: Our results suggest that nasal colonization with MRSA ST398 is a complex event that is accompanied with changes in bacterial gene expression regulation and metabolic adaptation.

Keywords: MRSA ST398, Microarray, Colonization, Ex vivo model

\section{Background}

Staphylococcus aureus is an opportunistic pathogen colonizing the upper respiratory tract and skin of humans and other mammalian species. The nose is considered to be the primary ecological niche of $S$. aureus in humans [1]. Nasal carriage of S. aureus has been identified as a risk factor for the development of various infections in humans [1].

In 2004 a new distinct sequence type (ST398) of methicillin-resistant $S$. aureus (MRSA) has been isolated

\footnotetext{
*Correspondence: b.duim@uu.nl

'Department of Infectious Diseases and Immunology, Faculty of Veterinary Medicine, Utrecht University, Utrecht, The Netherlands

${ }^{7}$ Department of Infectious Diseases and Immunology, Veterinary Faculty, Utrecht University, Yalelaan 1, PO BOX 80165, 3508 TD Utrecht, The Netherlands

Full list of author information is available at the end of the article
}

from pigs in the Netherlands [2]. Since then, MRSA ST398 has been detected in pigs, veal calves and poultry around the world [3-5]. The transmission of MRSA ST398 from livestock to humans has been reported in many countries $[6,7]$ and contact with livestock is recognized as a risk factor for human colonization $[4,8]$. Additionally, ST398 isolates may cause infections in humans [9]. However, the mechanisms underlying successful colonization of pigs and other livestock are incompletely understood.

The molecular mechanisms involved in S. aureus colonization have been mainly studied using human cell cultures [10] as well as rodent models [11,12]. S. aureus colonization involves many factors [13]. A crucial step of colonization is attachment to eukaryotic cells which involves several essential factors such as: clumping factor 
B (ClfB), iron-regulated surface determinant protein A (IsdA) and wall teichoic acid (WTA) [13]. Mutants deficient in one of the genes responsible for expressing these components displayed reduced cell-attachment properties in vitro [10] and showed reduced colonization in animal models [14-16]. Additionally, a ClfB mutant showed weakened colonization in the nares of human volunteers when compared to wild type bacteria, suggesting that ClfB is one of the main $S$. aureus factors involved in human colonization [17]. In addition to these factors, several other proteins (e.g., SdrC, SdrD, SasG, and FnbpA) appear to be involved in colonization by binding to desquamated nasal epithelial cells which confirms the multi-factorial nature of S. aureus-host interactions [18,19].

The natural occurrence of $S$. aureus in pigs may involve similar colonization factors as assumed for humans. On the other hand, it has been shown that the MRSA ST398 prevalence in pigs is high and that pigs are very rarely colonized by other $S$. aureus lineages. These observations suggest that additional factors may be involved in successful host adaptation and maintenance of colonization of S. aureus ST398 in livestock. A study of Viana et al. suggested that the presence of an additional von Willebrand binding factor protein (vWbp), encoded on a pathogenicity island (SaPI), represents a host adaptation factor of $S$. aureus for animals [20]. Moreover, it has been suggested that mobile genetic elements (MGEs) play a central role in the adaptation of bacteria to different host species [21].

To study S. aureus colonization in pigs, we have developed an ex vivo porcine nasal mucosa explants model [22]. Our aim was to identify bacterial factors involved in maintenance of $S$. aureus colonization in pigs by analyzing and documenting global gene expression changes in $S$. aureus during ex vivo colonization. This is the first study to examine changes in the complete $S$. aureus transcriptome during experimental colonization which mimics natural MRSA ST398 colonization in pigs.

\section{Results}

\section{Persistence of MRSA ST398 S0462 on porcine mucosa explants}

The ability of $S$. aureus to colonize porcine mucosa explants was defined as persistence or outgrowth of MRSA S0462 on the explants. The explants $\left(1 \mathrm{~cm}^{2}\right)$ were inoculated with $1 \mathrm{ml} 3 \times 10^{8} \mathrm{CFU} / \mathrm{ml}$. After $2 \mathrm{~h}$ of incubation $\left(37^{\circ} \mathrm{C}\right)$ and washing of the explants, approximately $8 \times 10^{6} \mathrm{CFU} / \mathrm{cm}^{2}$ (3\%) adhered to the explants. The presence of $S$. aureus S0462 on the mucosa explants was followed for an additional $180 \mathrm{~min}$. During the first $30 \mathrm{~min}$ of this latter period, MRSA S0462 showed an initial decline in the number of CFU to approximately $3 \times 10^{6}$. Bacterial presence remained stable until $90 \mathrm{~min}$ into the experiment. In the following $90 \mathrm{~min}$ a significant increase of tissue-associated bacteria to approximately
$4 \times 10^{7} \mathrm{CFU} / \mathrm{cm}^{2}$ was observed (Figure 1 ). This indicates that MRSA S0462 is able to establish and maintain colonization on the nasal explants during the experiment.

\section{S. aureus transcriptome dynamics during ex vivo colonization}

To identify genes that possibly contributed to the successful maintenance of colonization of S. aureus ST398 in pigs we analyzed the global changes in MRSA S0462 gene expression during ex vivo colonization on porcine nasal mucosa explants compared to time-point 0 (directly after removal of unbound bacteria). From MRSA S0462, recovered at different time-points from the explants, RNA was isolated, converted to cDNA and the cDNA hybridized with the $S$. aureus microarray.

In total we documented significant changes in the expression of 166 genes compared to time-point 0 . Transcripts that were significantly up- or down-regulated (adjusted $\mathrm{p}=0.05$ ) were visualized in a hierarchical clustering (Figure 2). Regulation of 148 of these 166 genes was deemed to be biologically relevant (at least 2 -fold linear change at least one time-point). A total of 75 genes were negatively regulated and 73 genes were positively regulated (Figure 2 and Additional file 1: Table S2). In general, there were no transcripts that changed in expression direction during the ex vivo colonization assay. For the majority of genes the change in expression compared $\mathrm{t}=0 \mathrm{~min}$ was maximal for $\mathrm{t}=60 \mathrm{~min}$, whereas fewer genes showed their maximum expression during $\mathrm{t}=30 \mathrm{~min}$ and $t=90 \mathrm{~min}$. Only a minority of the genes showed relatively large changes in expression for $\mathrm{t}=180 \mathrm{~min}$ compared to $\mathrm{t}=0 \mathrm{~min}$.

The regulated genes were mapped to available KEGG pathways based on the gene IDs. Most regulated genes

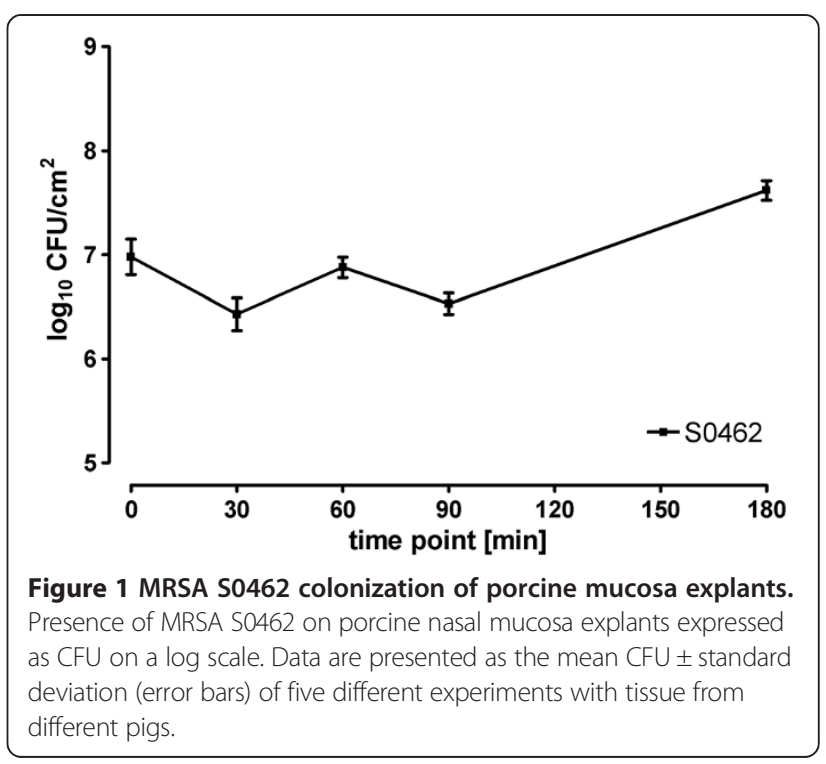




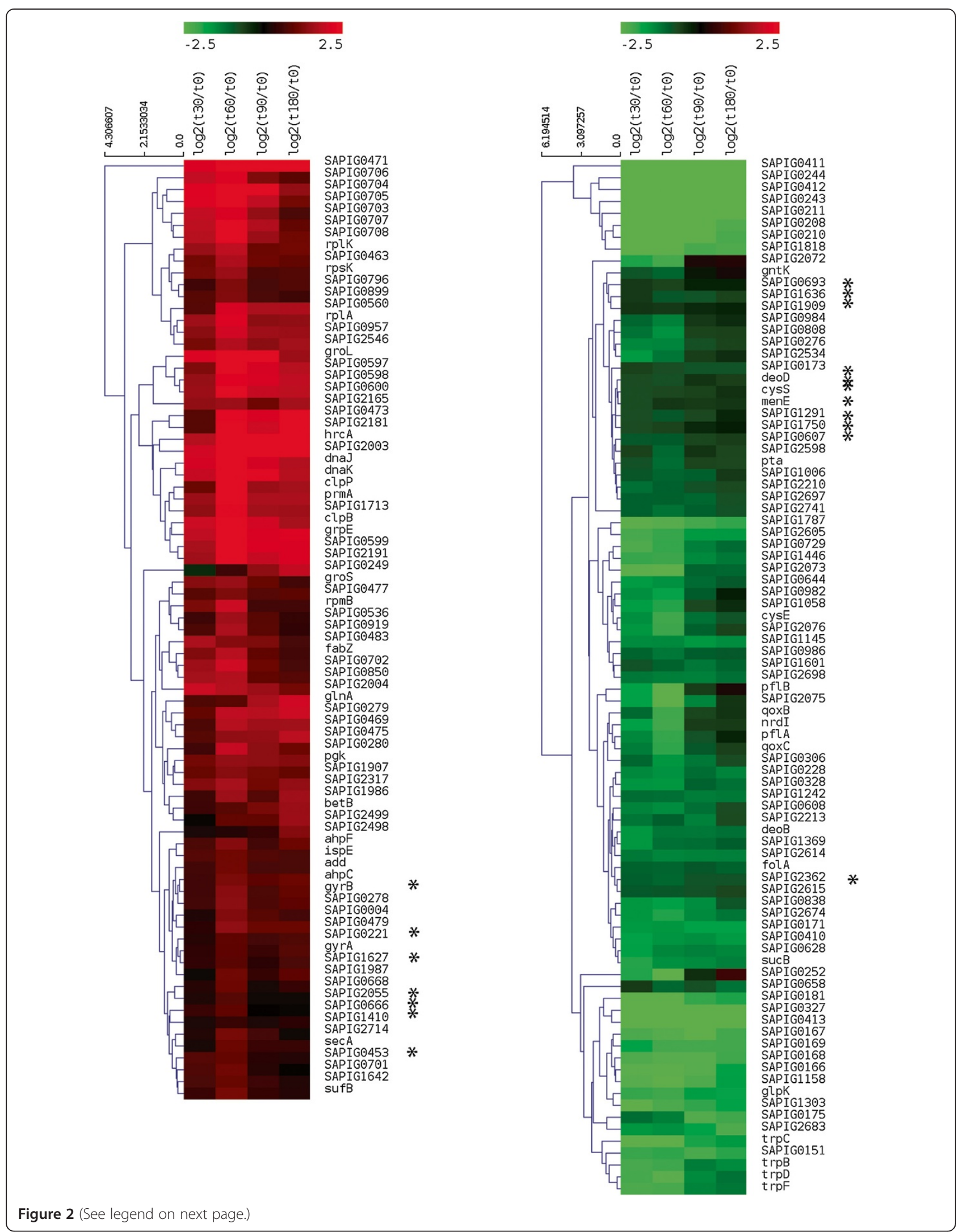


were involved in metabolic processes, probably reflecting metabolic adaptation to nasal mucosa explants, of fatty acid biosynthesis, oxidative phosphorylation, and of phenylalanine, tyrosine and tryptophan biosynthesis (Additional file 1: Table S2). In addition, regulation of the expression of genes that are part of the agr twocomponent regulatory system and a number of virulence genes was observed.

The genome of MRSA S0462 contains a vWbp-encoding pathogenicity island, SaPIbov5, which is widely distributed among $S$. aureus isolated from livestock [20]. Only a few genes located on this island were subject to regulation (int, $v w b p$ and some of the hypothetical proteins) in our model. It has been suggested that presence of the gene encoding an additional vWbP encoded on the pathogenicity island may play a role in bacterial adaptation to the animal host [20]. During ex vivo colonization $v w b p$ gene expression was up-regulated to its highest level at $t=60 \mathrm{~min}$ (3-fold change). Other genes located on the SaPIbov5 showed a larger change in expression after $\mathrm{t}=60 \mathrm{~min}$.

MRSA S0462 ex vivo colonization was also accompanied by changes in a number of putative virulence genes. The analysis showed 2.2 to 5.9-fold down-regulation of expression of genes in the cap operon encoding capsular biosynthesis during all time-points (2.2 to 5.5 -fold change) and of the hla gene conferring alpha-hemolysis -3.3 to -5.7 -fold change). Up-regulation was observed for the cysteine proteinase cluster $(\operatorname{scp} A B)$. The expression of the $\operatorname{sp} A$ gene encoding the cysteine proteinase was upregulated during the entire experiment (fold change varied from 4.2 to 11.5 ) and of the $s c p B$ encoding the cysteine proteinase cellular inhibitor (fold change varied from of 2.2 to 4.0). Moreover, the expression of the genes that constitute the agr locus, which encodes a quorum sensing system that controls the expression of virulence genes was also down-regulated mainly at $\mathrm{t}=60 \mathrm{~min}$ ( 4.2 to 6.9 -fold). However, regulation of agrD transcription was not detected.

To verify the microarray results, four genes of interest ( 2 up-regulated genes: $v w b p, s c p A$ and 2 down-regulated genes agrA, and $h l a$ ) were subjected to qRT-PCR (Figure 3) using the same RNA samples. The qRT-PCR results confirmed the microarray data $(v w b p \mathrm{r}=0.98, s c p A \mathrm{r}=0.93$, $\operatorname{agrA} \mathrm{r}=0.95$, and hla $\mathrm{r}=0.63$ ), although the fold change values determined by qRT-PCR were higher compared to the microarray data.

S. aureus ex vivo colonization did not show regulation of genes encoding surface proteins responsible for attachment of bacteria to the epithelium ( $c l f B$, is $d A$, and $f n b A$ ). To determine whether these genes were expressed during ex vivo colonization qRT-PCR analysis was performed. The results showed that the three genes important for human colonization: $\operatorname{clfB}$, is $d A$, and $f n b A$ were expressed during the colonization of the pig tissue (Additional file 2: Figure S1).

\section{Contribution of the vwbp and scpA to ex vivo colonization}

Next, we generated isogenic $v w b p$ and $s c p A$ deletion mutants of MRSA S0462 ( $\triangle v w b p$ and $\triangle s c p A)$ to investigate the potential role of these genes in MRSA S0462 colonization. The $v w b p$ and $s c p A$ mutants were tested in the ex vivo colonization assay (Additional file 3: Figure S2A and B). Surprisingly, the colonization pattern of the wild-type, $\Delta v w b p$ and $\Delta s c p A$ mutants did not show significant differences (Additional file 3: Figure S2C). These findings indicate that investigated genes are not crucial for colonization ex vivo, but may reflect adaptive changes during the colonization event.

\section{Discussion}

The nostrils are the primary reservoir of $S$. aureus both in humans and pigs. Nasal carriage of S. aureus has been identified as a risk factor for the development of various infections in humans [1]. During the last decade, swine have appeared as the major reservoir of MRSA $S$. aureus ST398 which also emerged in other livestock animals. Currently, contact with livestock is recognized as the main risk factor for MRSA ST398 colonization in humans. However, the factors which play a role in the colonization and maintenance of MRSA ST398 in livestock are unclear. $S$. aureus colonization in humans and rodent animal models is a multifactorial process which includes steps like bacterial attachment to the cells, immune escape as well as competition between $S$. aureus and natural flora. Identification of essential factors involved in $S$. aureus maintenance of colonization and adaptation in livestock are difficult to perform in an in vivo setting. Previously, we reported the successful establishment of an ex vivo model to study MRSA colonization [22]. Using this model we were able to mimic the natural situation in pig's noses under controlled conditions. During the ex vivo colonization, an initial decline in the number of CFU of S. aureus S0462 during the first $30 \mathrm{~min}$ after inoculation was observed, which indicated that some bacteria still detach from the mucosa or die. Later, a significant increase in the number of bacteria was observed, which 


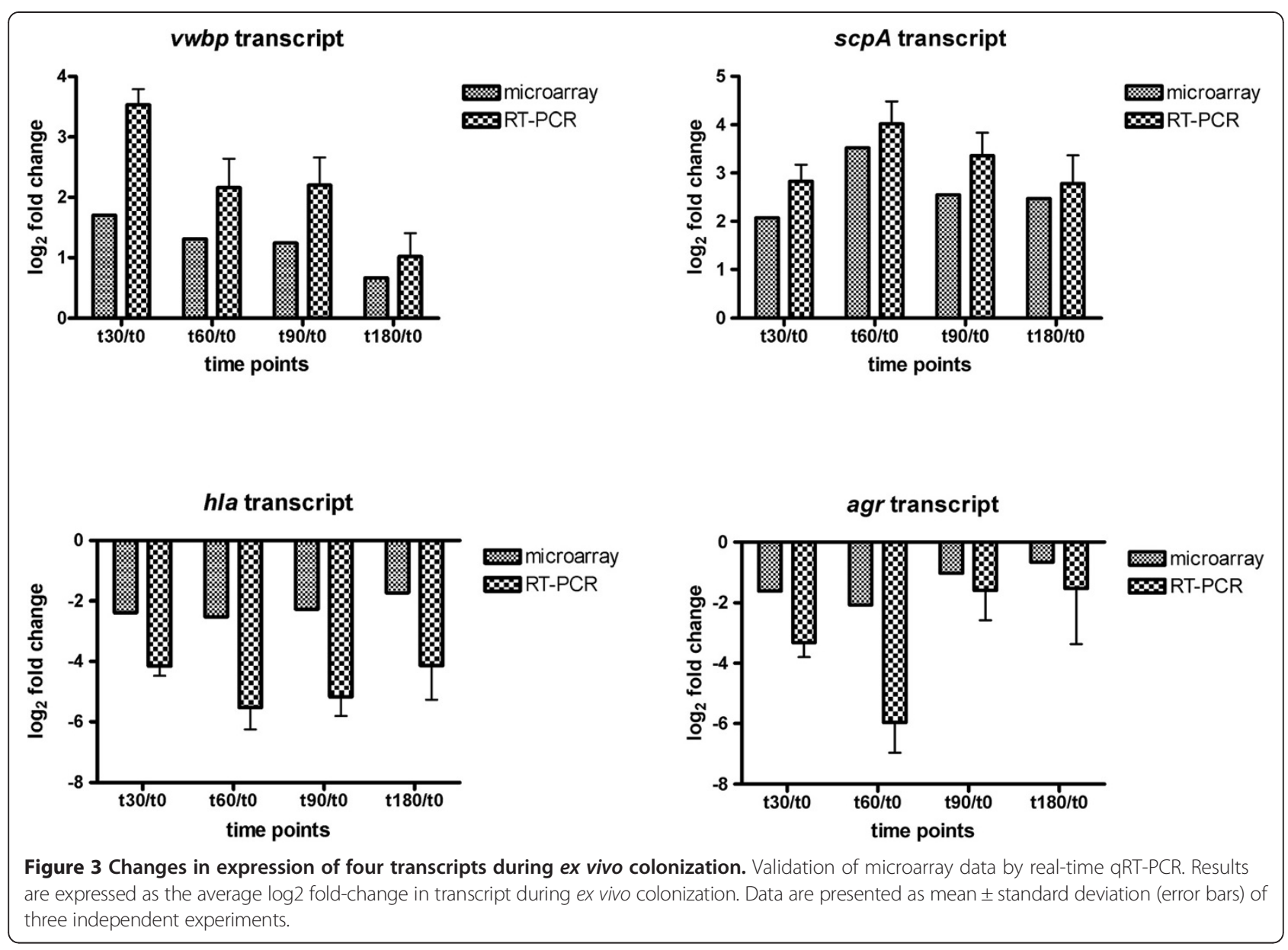

indicated maintenance of colonization and even outgrowth of the bacteria. From these data we concluded that establishment of $S$. aureus in our model mimics the initial phases of nasal colonization defined as maintenance and/or outgrowth.

Data on gene expression of $S$. aureus during colonization is limited to the direct transcript analysis of a few genes $[23,24]$. Global changes in gene expression during colonization have not been studied before. The major aim of our study was to identify bacterial factors involved in maintenance of colonization by determining and documenting the changes in S. aureus gene expression during ex vivo colonization.

The expression of 148 biologically relevant genes was regulated during culture on mucosa, when compared to $t=0$, the moment that unbound bacteria were removed from the explants. The expression of the majority of genes was highly regulated at $60 \mathrm{~min}$ after inoculation. At this time-point a net loss of bacteria is changing to a net increase mimicking colonization.

Based on at least a two-fold change in expression, MRSA colonization apparently resulted in changes in regulation of metabolic pathways. The biosynthesis pathways for phenylalanine, tyrosine and tryptophan biosynthesis pathways were down-regulated, in contrast to the genes involved in the main amino acid biosynthesis pathways which were not regulated in our system. The active protein biosynthesis, together with lack of regulation of the de novo amino acid biosynthesis pathways indicates that the bacterial cells have access to these amino acids. The ex vivo colonization was performed at an air-liquid interface, where bacteria may have had contact with the cultivation medium (RPMI and DMEM) containing free amino acids which may have served as a source of amino acids for bacteria, although we carefully avoided the presence of culture medium on top of the explants.

A crucial step of $S$. aureus nasal colonization is adhesion to epithelial cells. It has been shown that ClfB, IsdA proteins and WTA are essential factors for $S$. aureus adherence to human cell lines in vitro $[10,19]$, nasal colonization of rodents $[14,16,25]$, and for nasal colonization of humans [17]. After initial adherence, during the first 30 min MRSA S0462 showed an initial decline in the number of CFU and significant increase of tissue-associated bacteria in later phase of the experiment consistent with colonization. In the pig nasal ex vivo system we did not observe 
regulation of expression of the adhesion genes, however the genes were expressed. In our settings, bacteria used for tissue inoculation were harvested in the midlog phase, where adhesion factors are well expressed. Therefore, contact with the nasal epithelium did not result in regulation of the expression of genes encoding adherence factors such as $c l f B$, isd $A$, and $f n b A$. The alternative explanation, that the relevant mRNAs were not detected is unlikely based on the results of the data analysis and experience with highly similar microarray experiments for $S$. aureus [26].

Expression of the most adhesins is under the regulation of the agr system [23]. The agr system has a dual action in the global gene regulation in S. aureus. This system positively regulates toxins, extracellular proteases, immunomodulation factors and capsule biosynthesis, but represses the expression of some of the surface proteins such as protein A, coagulase and fibrinogen binding protein past the exponential phase. During ex vivo colonization the agr locus was down-regulated. However, the $\operatorname{agr} D$ gene, part of the agr locus, was not significantly regulated. This gene may be expressed at a basal level without a change in regulation. Additionally, we observed down-regulation of hla and the cap operon, which are controlled by the agr system (down-regulated). Burian et al. had similar findings showing that during $S$. aureus colonization of the human nose and in cotton rats the agr system is weakly expressed $[23,24]$.

Interestingly, the cysteine proteinase operon $(s c p A B)$ was up-regulated during the whole experiment. ScpA is known to cleave a number of extracellular matrix components and it has been suggested to play a role in bacterial migration from the sites of initial colonization [27,28], but it may also play a role in the acquisition of nutrients [29]. It has been suggested that the extracellular proteases can promote $S$. aureus skin and nares colonization by degradation of some $S$. aureus virulence factors like toxins [30]. Moreover, it has been shown that S. aureus ScpA protease is associated with diseases such as Staphylococcal Scalded Skin Syndrome [31] and is involved in vascular leakage causing sepsis [27]. The up-regulation of the extracellular proteinase ScpA indicates that this protein may be involved in establishment of colonization ex vivo. To study the influence of the cysteine proteinase for colonization ex vivo, we generated a ScpA isogenic mutant. However, a single knockout mutant did not show any phenotypic difference in colonization pattern ex vivo, which indicates that ScpA does not play a crucial role in colonization/ adaptation ex vivo.

Also the up-regulation of some genes located on SaPIbov5 was observed. This mobile element encodes an additional von Willebrand factor binding protein. It has been shown that $S$. aureus harboring a pathogenicity island with the additional $v w b p$ are widely distributed in ruminants and it has been suggested that this protein is one of the adaptation factors for $S$. aureus to animal hosts [20]. Our study showed that during S. aureus ST398 interaction with porcine nasal epithelium the $v w b p$ was strongly up-regulated during the first phase of the experiment $(\mathrm{t}=30 \mathrm{~min})$, where the initial decline of CFU was observed. This may indicate that $v w b p$ may be important in $S$. aureus adaptation to the porcine nasal epithelium and may promote $S$. aureus colonization ex vivo. However, the single knockout mutant did not show any phenotypic difference in colonization pattern ex vivo, which indicates that $v w b p$ also does not play a crucial role in colonization/ adaptation ex vivo.

In this study, we successful applied an ex vivo model to study changes in the gene expression regulation during colonization using one reference ST398 strain. Our previous study showed in an ex vivo model, colonization of different MRSA types from animal and human origin [22]. A study of Szabo et al. also reported a slight difference in pig colonization between different MRSA types [32]. Further study on gene expression of different $S$. aureus strains, during ex vivo colonization should be performed to understand strain specific colonization mechanisms.

There are few limitations of our model, which may influence $S$. aureus gene expression. First of all, adhesion of the bacteria onto the tissue was performed in DPBS. Furthermore, the host immune response will be absent, which might manifest itself in lack of regulation of the immune escape genes and influence $S$. aureus gene expression. Moreover, in our system we are not able to preserve the natural microbiota present in nasal mucosa tissue. In natural conditions, S. aureus must also compete with other bacterial species, which also would influence the global changes in gene expression. Recently, it has been reported that in humans, these interactions in the microbiota of the nasal cavity, influence the colonization of $S$. aureus [33]. Investigation on the microbiota in the nasal cavity of pigs and the bacteria interaction is necessary to understand the colonization process of $S$. aureus.

\section{Conclusions}

We could for the first time establish changes in the global gene expression pattern of $S$. aureus during ex vivo colonization. Additionally, this study suggests that nasal colonization with MRSA ST398 is a complex event that is accompanied with changes in bacterial gene expression regulation and metabolic adaptation.

\section{Methods}

\section{Ethics statement}

Tissue was isolated from pigs that were euthanized after a cardiovascular study at the UMCU (Utrecht, the Netherlands), which was approved by the Utrecht Animal Ethics Committee in accordance with article 18 of the 
Dutch Experiments on Animals Act and carried out in accordance with the European Guidelines for the accommodation and care of animals used for experimental and other scientific purposes as laid down in EU Recommendation 2007/526/EC.

\section{Bacterial strain and ex vivo colonization assay}

MRSA ST398 S0462 strain (spa-type: t011, SCCmec IV) was taken for the transcription experiments because in a previous study it was shown to adhere to and grow on pig nasal explants. S0462 was isolated from a colonized pig. The strain was sequenced and the sequence was submitted to EMBL (accession numbers CAVX010000001CAVX010000134) [34]. The preparation of nasal mucosa explants from pigs was performed as previously described [22]. The explants were inoculated with MRSA isolates as described previously [22]. Briefly, S. aureus strains were grown overnight in $\mathrm{BHI}$ at $37^{\circ} \mathrm{C}$. A $2 \%$ aliquot was inoculated into fresh $10 \mathrm{ml}$ broth and grown at $37^{\circ} \mathrm{C}$ under shaking $(200 \mathrm{rpm})$ to mid-exponential phase (approximately $4 \mathrm{~h}$ ). Bacteria were harvested by centrifugation at $3,750 \times g$ for $5 \mathrm{~min}$, washed 3 times in Dulbecco's Phosphate-Buffered Saline (DPBS), and suspended at an $\mathrm{OD}_{600}$ of 0.6 (approximately $3 \times 10^{8} \mathrm{CFU} / \mathrm{ml}$ ) in DPBS. The explants were inoculated with $1 \mathrm{ml}$ of bacterial suspension in DPBS (approximately $3 \times 10^{8}$ colony forming units $(\mathrm{CFU}) / \mathrm{ml}$ in DPBS) for $2 \mathrm{~h}$ to allow the bacteria to adhere to the tissue (adhering time). Next, bacteria were washed with DPBS to remove unbound bacteria and explants were cultivated at $37^{\circ} \mathrm{C}$ in a $5 \% \mathrm{CO}_{2}$ atmosphere. At different time-points (0, 30, 60, 90, and $180 \mathrm{~min})$ after removal of unbound bacteria, samples for RNA extraction and subsequent transcription analysis were taken. At these time-points explants were washed three times with $1 \mathrm{ml}$ DPBS. Bacteria were isolated from the explants by scraping the epithelium surface using cell scrapers (Falcon, Becton Dickinson, The Netherlands) and resuspended in $1 \mathrm{ml}$ of DPBS with $0.1 \%$ Triton X-100. Nine hundred $\mu \mathrm{l}$ of the bacterial suspension was immediately centrifuged at $20,000 \times g$ for $2 \mathrm{~min}$ (room temperature) and the resulting pellet was frozen at $-80^{\circ} \mathrm{C}$ before RNA isolation. The remaining $100 \mu \mathrm{l}$ of bacterial suspension was serially diluted in DPBS and plated on blood agar plates (Oxoid, UK). The plates were incubated overnight at $37^{\circ} \mathrm{C}$ and CFU were enumerated after $24 \mathrm{~h}$. The colonization assay was repeated independently four times. Additionally, to investigate MRSA adaptation to mucosa, the suspension was incubated using the same growth conditions, but without contact with an explant. After $2 \mathrm{~h}$ bacteria were collected for RNA isolation.

\section{RNA extraction}

RNA was purified using the NucleoSpin RNA II total RNA isolation kit (Macherey-Nagel, Germany) according to manufacturer's protocol with some adjustments as described [26]. No removal of eukaryotic RNA was performed as the specificity of the microarray was high enough to discriminate between different $S$. aureus strains.

\section{Microarray design}

The microarray was specifically developed for multiple $S$. aureus strains. The complete design was performed in a two-step procedure. First, 60-mer oligonucleotides were designed each 40 base pairs on alternating strands for the first sequence, ST398. Second, for each next sequence oligonucleotides were only designed for regions that were not probed by previously designed oligonucleotides. Oligonucleotides that match a sequence with a bitscore over 80 were considered as usable for probing. Using these parameters 121,901 probes were generated and manufactured as a microarray by Nimblegen (Roche) in a $12 \times 135 \mathrm{~K}$ format.

For more details see the Additional file 4.

\section{Labeling of total RNA, hybridization and scanning}

Total RNA was labeled with fluorescent dyes by an amplification procedure and direct labeling. A total of $100 \mathrm{ng}$ RNA was used as input for the Ovation Pico WTA System according to manufacturer's instructions (Nugen Technologies, Inc, USA). Two $\mu \mathrm{g}$ of purified and amplified cDNA was used as input for labeling by randomly priming with Superscript II reverse transcriptase (Invitrogen, The Netherlands), random octamers $(100 \mathrm{ng} / \mu \mathrm{l})$ and actinomycine $\mathrm{D}$, in a total volume of $10 \mu \mathrm{l}$, for $2 \mathrm{~h}$ at $42^{\circ} \mathrm{C}$ with the incorporation of Cy5- or Cy3-dUTP (Amersham, USA) with a ratio dUTP/dTTP of 3/1. Labeled cDNA was purified using Qia-quick PCR purification kit (Qiagen, USA). Incorporation of Cy3 or Cy5 was determined using a NanoDrop ND-1000.

The common reference was created by pooling Cy5labeled RNA samples. Labeled cDNA was hybridized according to manufacturer's protocol (Roche NimbleGen, The Netherlands). A total of $1.1 \mu \mathrm{g}$ Cy3-labeled cDNA and $1.1 \mu \mathrm{g}$ Cy5-labeled common reference was mixed in $7.2 \mu \mathrm{l}$ of NimbleGen hybridization cocktail. The mixture was heated to $65^{\circ} \mathrm{C}$ for $5 \mathrm{~min}$, and a total of $6 \mu \mathrm{l}$ was loaded onto the custom made $S$. aureus array and hybridized for $18 \mathrm{~h}$ at $42^{\circ} \mathrm{C}$ in a dedicated hybridization chamber (Roche NimbleGen, The Netherlands).

After the hybridization the arrays were dismantled at $42^{\circ} \mathrm{C}$ and washed in buffer 1 for $2 \mathrm{~min}$ at room temperature, then $1 \mathrm{~min}$ in wash buffer 2 at room temperature and finally $15 \mathrm{sec}$ in wash buffer 3 at room temperature (Roche NimbleGen, The Netherlands). Slides were spun for $30 \mathrm{sec}$ at $300 \mathrm{rpm}$ to dry and scanned immediately in an Agilent DNA MicroArray Scanner. Data was extracted and processed using NimbleScantm software (version 2.6, Roche NimbleGen, The Netherlands). 


\section{Data analysis and statistical analyses}

Processing of the data was performed using $\mathrm{R}$ (version 2.14.1) and the Bioconductor MAANOVA package (version 1.10.0). All slides were subjected to a set of quality control checks, which consisted of visual inspection of the scans, examination of the consistency among the replicated samples by principal component analysis (PCA), testing against criteria for signal to noise ratios, testing for consistent performance of the labeling dyes and visual inspection of pre- and post-normalized data with box and ratio-intensity plots. After log2 transformation, the data were normalized by a within-slide LOWESS smoothing procedure. The features were annotated for S. aureus ST398 using GenBank, and gene expression values were calculated using the robust multi-array average (RMA) algorithm [35], which also performs between slide normalization. The resulting data were analyzed using an ANOVA model. A contrast analysis was applied to compare the samples from each time point with the control group $(t=0)$ [36]. For hypothesis testing a permutation based Fs test was used [37] and the resulting $\mathrm{P}$-values were corrected for false discoveries according to Storey and Tibshirani [38]. The significance threshold was set at 0.05 FDR. A complete set of the microarray data has been deposited at the GEO database (accession number GSE47910). Changes in expression of at least 2-fold linear change were arbitrarily considered biologically relevant.

For more details see the Additional file 4 and Jonker et al. [39].

\section{Real-time quantitative reverse transcriptase PCR}

RNA samples used in the microarray experiments were also analyzed by quantitative reverse-transcriptase realtime PCR (qRT-PCR). Purified S. aureus RNA was converted using the Bacterial $\mathrm{H}-\mathrm{TR}$ cDNA synthesis kit (AmpTech, Germany). The cDNA products were subsequently used in qRT-PCR using SYBR Green Master Mix for qRT-PCR (Takara Bio Inc, Japan) according to the manufacturer's instruction and the qRT-PCR reaction was performed in a LightCycler 480 (Roche, The Netherlands). The transcripts for $v w b p, s c p A, h l y$ and $a g r$ were amplified using primers listed in Additional file 5: Table S1. All signals were normalized to $\operatorname{aro} E$ and gmk gene transcripts (both housekeeping genes) [40]. Relative quantification of gene expression was calculated using the comparative cycle threshold method as described previously by Livak and Schmittgen [41]. Data obtained are expressed as the mean $\log _{2}$ fold-change in transcript during colonization ex vivo for selected post time-points after removal of unbound bacteria (30, 60, 90 , and $180 \mathrm{~min}$ ) relative to the $\mathrm{t}=0$ control sample. All time-points were tested in triplicate.

\section{Construction of an isogenic $v w b p$ and $s c p A$ deletion strain} ( $\triangle v w b p$ and $\triangle s c p A$ )

Isogenic $v w b p$ and $s c p A$ deletions in the S0462 strain were generated by allelic replacement as described [42] with a slight modification. Briefly, regions around 1000 bp flanking the $v w b p$ and $s c p A$ locus were amplified by PCR using primers listed in Additional file 5: Table S1. The resulting PCR fragments were used as a template to create an insertion fragment by PCR overlap. Next, the PCR fragment was digested using EcoRI and NotI restriction endonucleases (Fermentas, Lithuania) and cloned into pKOR1 vector. The ligation product was transformed into competent Escherichia coli DC10b cells [43] and grown on LB agar containing $100 \mu \mathrm{g} / \mathrm{ml}$ ampicillin. Purified plasmid (500 ng) containing the correct insert (confirmed by sequencing) was electroporated into the target strain, S. aureus S0462, using the settings: $200 \Omega, 25 \mu \mathrm{F}$ and $1.5 \mathrm{kV}$. After electroshock $200 \mu \mathrm{l}$ of TSB was added and cells were incubated at $37^{\circ} \mathrm{C}$ for $1 \mathrm{~h}$ with shaking, to allow recovery. The cells were plated on TSA plates containing $7.5 \mu \mathrm{g} / \mathrm{ml}$ chloramphenicol and grown overnight at $30^{\circ} \mathrm{C}$. Single colonies were grown in TSB overnight at $30^{\circ} \mathrm{C}$ with vigorous shaking. Plasmid integration was checked by overnight culture at $43^{\circ} \mathrm{C}$ on TSA with $7.5 \mu \mathrm{g} / \mathrm{ml}$ chloramphenicol. Colonies were screened for single cross-over by PCR. Single crossover mutants were grown in TSB without antibiotic at $30^{\circ} \mathrm{C}$ overnight, diluted $1: 100,000$ in sterile water and $100 \mu \mathrm{l}$ was spread on TSA plate containing $50 \mu \mathrm{g} / \mathrm{ml}$ anhydrotetracycline and incubated at $37^{\circ} \mathrm{C}$ overnight. Large colonies were picked and cultured overnight at $37^{\circ} \mathrm{C}$ on TSA with $10 \mu \mathrm{g} / \mathrm{ml}$ chloramphenicol and plain TSA. Colonies growing only on plain TSA were assumed to be knock-out mutants. Putative mutants were validated by PCR amplification and sequencing of genomic DNA flanking the deletion. Confirmed knock-out strains were used in subsequent experiments.

\section{Availability of supporting data}

The microarray data has been deposited at the GEO database (accession number GSE47910). http://www.ncbi.nlm. nih.gov/geo/query/acc.cgi?token $=x z o j x w q w u i a q q r u \& a c c=$ GSE47910.

\section{Additional files}

Additional file 1: Table S2. Changes in the MRSA S0462 transcriptome during ex vivo colonization. Microarray results are presented as the mean linear fold-change from six separate experiments. Only transcripts with significant fold-change ( $p$ value $<0.05$-fold change in transcriptome) are included. The results are expressed as the average linear fold-change in transcript during ex vivo colonization compared to $t=0$.

Additional file 2: Figure S1. Expression of three important colonization genes: $C l f B$, is $d A$, and $f n b A$ during ex vivo colonization. The qRT-PCR 
results are expressed as the average $\log 2$ fold-change in transcript during ex vivo colonization compared to $t=0$.

Additional file 3: Figure S2. Construction and characterization of a knockout vwb and scpA strains in MRSA S0462. A) Schematic representation of the deletion $v w b$ or $s c p A$ genes in MRSA S0462 strain. B) PCR confirmation of $v w b$ and scpA deletion mutagenesis. C) MRSA S0462 wild-type, $\Delta v w b$, and $\triangle s c p A$ colonization of porcine mucosa explants. Data are presented is the mean log CFU \pm standard deviation (error bars) of three different pig experiments.

\section{Additional file 4: Supporting information.}

Additional file 5: Table S1. Primers, plasmids and strains used in this study.

\section{Competing interest}

The authors declare that they have no competing interests.

\section{Authors' contributions}

$\mathrm{PT}, \mathrm{BD}, \mathrm{AF}$, JW conceived this study. PT carried out the analysis and wrote the manuscript. FW, MJ conducted the array analysis. BD, AF, JW, TB and JP were involved in discussion of the work and manuscript revision. All authors read and approved the final version.

\section{Acknowledgments}

The authors would like to thank Evelyn Velema from Department of Experimental Cardiology of University Medical Center Utrecht and Louis van den Boom from the Faculty of Veterinary Medicine at Utrecht University for helping with mucosa explants isolation. We are grateful to Suzanne H. M. Rooijakkers and Marc D. Jansen from Department of Medical Microbiology of University Medical Center Utrecht for support in generating knockout mutants.

\section{Author details}

'Department of Infectious Diseases and Immunology, Faculty of Veterinary Medicine, Utrecht University, Utrecht, The Netherlands. ${ }^{2}$ MicroArray Department, University of Amsterdam, Amsterdam, The Netherlands. ${ }^{3}$ Central Veterinary Institute of Wageningen UR, Lelystad, The Netherlands. ${ }^{4}$ Department of Medical Microbiology, University Medical Center Utrecht, Utrecht, The Netherlands. ${ }^{5}$ Currently at the Department of Bionanoscience, Delft University of Technology, Delft, The Netherlands. ${ }^{6}$ Currently at the Department of Technology, Leiden University of Applied Sciences, Leiden, The Netherlands. ${ }^{7}$ Department of Infectious Diseases and Immunology, Veterinary Faculty, Utrecht University, Yalelaan 1, PO BOX 80165, 3508 TD Utrecht, The Netherlands.

\section{Received: 22 July 2014 Accepted: 13 October 2014}

Published: 20 October 2014

\section{References}

1. Wertheim HF, Melles DC, Vos MC, van Leeuwen W, van Belkum A, Verbrugh HA Nouwen JL: The role of nasal carriage in Staphylococcus aureus infections. Lancet Infect Dis 2005, 5:751-762.

2. Voss A, Loeffen F, Bakker J, Klaassen C, Wulf M: Methicillin-resistant Staphylococcus aureus in pig farming. Emerg Infect Dis 2005, 11:1965-1966.

3. Smith TC, Pearson N: The emergence of Staphylococcus aureus ST398. Vector borne and Zoonotic Diseases 2010, 11(4):327-339.

4. Graveland H, Wagenaar JA, Bergs K, Heesterbeek H. Heederik D: Persistence of livestock associated MRSA CC398 in humans is dependent on intensity of animal contact. PLoS One 2011, 6:e16830.

5. Graveland H, Duim B, van Duijkeren E, Heederik D, Wagenaar JA: Livestock-associated methicillin-resistant Staphylococcus aureus in animals and humans. Int J Med Microbiol 2011, 301:630-634.

6. van Cleef BA, Monnet DL, Voss A, Krziwanek K, Allerberger F, Struelens M, Zemlickova H, Skov RL, Vuopio-Varkila J, Cuny C, Friedrich A, Spiliopoulou I, Paszti J, Hardardottir H, Rossney A, Pan A, Pantosi A, Borg M, Grundmann H, Mueller-Premru M, Olsson-Liljequist B, Widmer A, Harbarth S, Schweiger A, Unal S, Kluytmans JA: Livestock-associated methicillin-resistant Staphylococcus aureus in humans, Europe. Emerg Infect Dis 2011, 17:502-505.

7. Golding GR, Bryden L, Levett PN, McDonald RR, Wong A, Wylie J, Graham MR, Tyler S, Van Domselaar G, Simor AE, Gravel D, Mulvey MR: Livestock-associated methicillin-resistant Staphylococcus aureus sequence type 398 in humans, Canada. Emerg Infect Dis 2010, 16:587-594

8. van den Broek IV, van Cleef BA, Haenen A, Broens EM, van der Wolf PJ, van den Broek MJ, Huijsdens XW, Kluytmans JA, van de Giessen AW, Tiemersma EW: Methicillin-resistant Staphylococcus aureus in people living and working in pig farms. Epidemiol Infect 2009, 137:700-708.

9. van Belkum A, Melles DC, Peeters JK, van Leeuwen WB, van Duijkeren E, Huijsdens XW, Spalburg E, de Neeling AJ, Verbrugh HA, Dutch Working Party on S, Research of M-S: Methicillin-resistant and -susceptible Staphylococcus aureus sequence type 398 in pigs and humans. Emerg Infect Dis 2008, 14:479-483.

10. O'Brien LM, Walsh EJ, Massey RC, Peacock SJ, Foster TJ: Staphylococcus aureus clumping factor $\mathrm{B}$ (ClfB) promotes adherence to human type cytokeratin 10: implications for nasal colonization. Cell Microbiol 2002, 4:759-770

11. Kiser KB, Cantey-Kiser JM, Lee JC: Development and characterization of a Staphylococcus aureus nasal colonization model in mice. Infect Immun 1999, 67:5001-5006.

12. Kokai-Kun JF: The Cotton Rat as a model for Staphylococcus aureus nasal colonization in humans: cotton rat S. aureus nasal colonization model. Method Mol Biol 2008, 431:241-254.

13. Edwards AM, Massey RC, Clarke SR: Molecular mechanisms of Staphylococcus aureus nasopharyngeal colonization. Mol Oral Microbiol 2012, 27:1-10.

14. Schaffer AC, Solinga RM, Cocchiaro J, Portoles M, Kiser KB, Risley A, Randall SM, Valtulina V, Speziale P, Walsh E, Foster T, Lee JC: Immunization with Staphylococcus aureus clumping factor B, a major determinant in nasal carriage, reduces nasal colonization in a murine model. Infect Immun 2006, 74:2145-2153.

15. Clarke SR, Brummell KJ, Horsburgh MJ, McDowell PW, Mohamad SA Stapleton MR, Acevedo J, Read RC, Day NP, Peacock SJ, Mond JJ, Kokai-Kun JF, Foster SJ: Identification of in vivo-expressed antigens of Staphylococcus aureus and their use in vaccinations for protection against nasal carriage. $J$ Infect Dis 2006, 193:1098-1108.

16. Weidenmaier C, Kokai-Kun JF, Kristian SA, Chanturiya T, Kalbacher H, Gross M, Nicholson G, Neumeister B, Mond JJ, Peschel A: Role of teichoic acids in Staphylococcus aureus nasal colonization, a major risk factor in nosocomial infections. Nat Med 2004, 10:243-245.

17. Wertheim HF, Walsh E, Choudhurry R, Melles DC, Boelens HA, Miajlovic H, Verbrugh HA, Foster T, van Belkum A: Key role for clumping factor B in Staphylococcus aureus nasal colonization of humans. PLoS Med 2008, 5:e17.

18. Roche FM, Meehan M, Foster TJ: The Staphylococcus aureus surface protein Sas $G$ and its homologues promote bacterial adherence to human desquamated nasal epithelial cells. Microbiology 2003, 149:2759-2767.

19. Corrigan RM, Miajlovic H, Foster TJ: Surface proteins that promote adherence of Staphylococcus aureus to human desquamated nasal epithelial cells. BMC Microbiol 2009, 9:22.

20. Viana D, Blanco J, Tormo-Mas MA, Selva L, Guinane CM, Baselga R, Corpa J, Lasa I, Novick RP, Fitzgerald JR, Penades JR: Adaptation of Staphylococcus aureus to ruminant and equine hosts involves SaPI-carried variants of von Willebrand factor-binding protein. Mol Microbiol 2010, 77:1583-1594.

21. McCarthy AJ, Witney AA, Gould KA, Moodley A, Guardabassi L, Voss A, Denis O, Broens EM, Hinds J, Lindsay JA: The distribution of mobile genetic elements (MGEs) in MRSA CC398 is associated with both host and country. Genome Biol Evol 2011, 3:1164-1174.

22. Tulinski P, Fluit AC, van Putten JPM, de Bruin A, Glorieux S, Wagenaar JA, Duim B: An ex vivo porcine pasal mucosa explants model to study MRSA colonization. PLoS One 2013, 8:e53783.

23. Burian M, Rautenberg M, Kohler T, Fritz M, Krismer B, Unger C, Hoffmann WH, Peschel A, Wolz C, Goerke C: Temporal expression of adhesion factors and activity of global regulators during establishment of Staphylococcus aureus nasal colonization. J Infect Dis 2010, 201:1414-1421.

24. Burian M, Wolz C, Goerke C: Regulatory adaptation of Staphylococcus aureus during nasal colonization of humans. PLoS One 2010, 5:e10040.

25. Clarke SR, Wiltshire MD, Foster SJ: IsdA of Staphylococcus aureus is a broad spectrum, iron-regulated adhesin. Mol Microbiol 2004, 51:1509-1519.

26. ten Broeke-Smits NJ, Pronk TE, Jongerius I, Bruning O, Wittink FR, Breit TM, van Strijp JA, Fluit AC, Boel CH: Operon structure of Staphylococcus aureus. Nucleic Acids Res 2010, 38:3263-3274.

27. Imamura T, Tanase S, Szmyd G, Kozik A, Travis J, Potempa J: Induction of vascular leakage through release of bradykinin and a novel kinin by 
cysteine proteinases from Staphylococcus aureus. J Exp Med 2005, 201:1669-1676.

28. Ohbayashi T, Irie A, Murakami Y, Nowak M, Potempa J, Nishimura Y, Shinohara M, Imamura T: Degradation of fibrinogen and collagen by staphopains, cysteine proteases released from Staphylococcus aureus. Microbiology 2011, 157:786-792

29. Travis J, Potempa J, Maeda H: Are bacterial proteinases pathogenic factors? Trends Microbiol 1995, 3:405-407.

30. Lindsay JA, Foster SJ: Interactive regulatory pathways control virulence determinant production and stability in response to environmental conditions in Staphylococcus aureus. Mol Gen Genet 1999, 262:323-331.

31. Amagai M, Matsuyoshi N, Wang ZH, Andl C, Stanley JR: Toxin in bullous impetigo and staphylococcal scalded-skin syndrome targets desmoglein 1. Nat Med 2000, 6:1275-1277.

32. Szabo I, Beck B, Friese A, Fetsch A, Tenhagen BA, Roesler U: Colonization kinetics of different methicillin-resistant Staphylococcus aureus sequence types in pigs and host susceptibilities. Appl Environ Microbiol 2012, 78:541-548.

33. Yan M, Pamp SJ, Fukuyama J, Hwang PH, Cho DY, Holmes S, Relman DA Nasal microenvironments and interspecific interactions influence nasal microbiota complexity and S. aureus carriage. Cell Host Microbe 2013, 14:631-640.

34. Schijffelen M, Konstantinov SR, Lina G, Spiliopoulou I, van Duijkeren E, Brouwer EC, Fluit AC: Whole genome analysis of epidemiologically closely related Staphylococcus aureus isolates. PLoS One 2013, 8:e78340

35. Irizarry RA, Hobbs B, Collin F, Beazer-Barclay YD, Antonellis KJ, Scherf $U$, Speed TP: Exploration, normalization, and summaries of high density oligonucleotide array probe level data. Biostatistics 2003, 4:249-264.

36. Smyth GK: Linear models and empirical bayes methods for assessing differential expression in microarray experiments. Stat Appl Genet Mol Biol 2004, 3:Article 3. PMID: 16646809.

37. Cui X, Hwang JT, Qiu J, Blades NJ, Churchill GA: Improved statistical tests for differential gene expression by shrinking variance components estimates. Biostatistics 2005, 6:59-75.

38. Storey JD, Tibshirani R: Statistical significance for genomewide studies. Proc Natl Acad Sci U S A 2003, 100:9440-9445.

39. Jonker MJ, de Leeuw WC, Marinkovic M, Wittink FR, Rauwerda H, Bruning O, Ensink WA, Fluit AC, Boel CH, Jong M, Breit TM: Absence/presence calling in microarray-based CGH experiments with non-model organisms. Nucleic Acids Res 2014, 42:e94.

40. Hirschhausen N, Schlesier T, Peters G, Heilmann C: Characterization of the modular design of the autolysin/adhesin Aaa from Staphylococcus aureus. PLoS One 2012, 7:e40353.

41. Livak KJ, Schmittgen TD: Analysis of relative gene expression data using real-time quantitative PCR and the 2(-Delta Delta $C(T)$ ) Method. Method 2001, 25:402-408.

42. Bae T, Schneewind O: Allelic replacement in Staphylococcus aureus with inducible counter-selection. Plasmid 2006, 55:58-63.

43. Monk IR, Shah IM, Xu M, Tan MW, Foster TJ: Transforming the untransformable: application of direct transformation to manipulate genetically Staphylococcus aureus and Staphylococcus epidermidis. mBio 2012, 3:10. doi:1128/mBio.00277-00211. Print 02012.

doi:10.1186/1471-2164-15-915

Cite this article as: Tulinski et al:: Staphylococcus aureus ST398 gene expression profiling during ex vivo colonization of porcine nasal epithelium. BMC Genomics 2014 15:915.

\section{Submit your next manuscript to BioMed Central and take full advantage of:}

- Convenient online submission

- Thorough peer review

- No space constraints or color figure charges

- Immediate publication on acceptance

- Inclusion in PubMed, CAS, Scopus and Google Scholar

- Research which is freely available for redistribution 